\title{
Late-life depression, heart failure and frontal white matter hyperintensity: a structural magnetic resonance imaging study
}

J.R.C. Almeida ${ }^{1}$,

T.C.T.F. Alves ${ }^{1}$,

M. Wajngarten ${ }^{2}$,

J. Rays ${ }^{2}$, C.C. Castro ${ }^{3}$,

Q. Cordeiro ${ }^{1}$, R.M.S. Telles ${ }^{1}$,

R.J. Fraguas ${ }^{1}$

and G.F. Busatto

\author{
${ }^{1}$ Instituto de Psiquiatria, ${ }^{2}$ Instituto do Coração, \\ Departamento de Cardiogeriatria, and \\ ${ }^{3}$ Instituto de Radiologia, Faculdade de Medicina, \\ Universidade de São Paulo, São Paulo, SP, Brasil
}

Correspondence

J.R.C. Almeida

Centro de Medicina Nuclear

Trav. Dr. Ovidio P. de Campos, s/n

05403-010 São Paulo, SP

Brasil

Fax: +55-11-3082-9223

E-mail: jca@websolute.com.br

Research supported by FAPESP

(No. 99/11562-0).

$\ldots \ldots \ldots \ldots \ldots \ldots \ldots$

Received July 29, 2004

Accepted November 30, 2004

\begin{abstract}
The relevance of the relationship between cardiac disease and depressive symptoms is well established. White matter hyperintensity, a bright signal area in the brain on T2-weighted magnetic resonance imaging scans, has been separately associated with cardiovascular risk factors, cardiac disease and late-life depression. However, no study has directly investigated the association between heart failure, major depressive symptoms and the presence of hyperintensities. Using a visual assessment scale, we have investigated the frequency and severity of white matter hyperintensities identified by magnetic resonance imaging in eight patients with late-life depression and heart failure, ten patients with heart failure without depression, and fourteen healthy elderly volunteers. Since the frontal lobe has been the proposed site for the preferential location of white matter hyperintensities in patients with late-life depression, we focused our investigation specifically on this brain region. Although there were no significant group differences in white matter hyperintensities in the frontal region, a significant direct correlation emerged between the severity of frontal periventricular white matter hyperintensity and scores on the Hamilton scale for depression in the group with heart failure and depression ( $\mathrm{P}=0.016$, controlled for the confounding influence of age). There were no significant findings in any other areas of the brain. This pattern of results adds support to a relationship between cardiovascular risk factors and depressive symptoms, and provides preliminary evidence that the presence of white matter hyperintensities specifically in frontal regions may contribute to the severity of depressive symptoms in cardiac disease.
\end{abstract}

Key words

- Major depressive disorder - Old age

- Magnetic resonance imaging

- Cardiac failure

- Cerebrovascular disease

- Cardiovascular risk factors 
Recently, the association between cardiovascular problems and major depression in elderly patients has been a subject of considerable interest. Approximately 20\% of individuals with heart failure (HF), acute myocardial infarction or recent cardiac catheterization also present significant depressive symptoms (1). HF is among the conditions associated with the largest number of inpatient admissions and public health costs. The presence of depressive symptoms in HF subjects predicts longer duration of in-hospital stays and higher frequency of outpatient medical visits after discharge compared to HF without concomitant depression (2).

The neurobiological mechanisms underlying the association between depressive symptoms and cardiac disease have not been clarified, but there are hypotheses that vascular abnormalities could play a significant role (1-4). One of the research areas that may help to clarify this issue is the investigation of white matter hyperintensities (WMH) in the brain. These areas of bright signal, best seen on T2-weighted magnetic resonance imaging (MRI) scans of the brain, are commonly associated with advanced age $(1,4)$. There have also been reports of increased frequency of $\mathrm{WMH}$ in the general population in association with cardiovascular risk factors and cardiovascular disease (4-7). Furthermore, there have been data indicating a higher incidence of WMH in samples of patients with major depressive symptoms emerging late in life. These findings have provided support to the concept of a "vascular" type of depression specifically referring to a subgroup of late-life depressive episodes with distinct clinical features and a poor outcome $(8,9)$.

In subjects with late-onset depression, there is evidence that WMH are predominantly located in brain regions seen as critical for the regulation of mood, particularly frontostriatal circuits $(3,4)$. These data are consistent with findings of frontal lobe impairment in biochemical (10), anatomical
(11) and histological (9) investigations in the brains of patient with a history of depressive disorders $(3,12)$.

Although WMH have been independently associated with the presence of cardiovascular disease and the emergence of depression in elderly subjects, no study has demonstrated to date the existence of a relationship between HF, major depression and WMH. In the present study, we investigated the presence of WMH in a group of HF subjects with co-morbid major depressive disorder, a group of non-depressive HF subjects, and a control group of healthy elderly volunteers in order to test the hypothesis that WMH in frontal regions may be associated with the presence of depressive symptoms in HF patients. This hypothesis was tested in two ways: by comparing the frequency and severity of WMH between the three groups, and by investigating the presence of significant correlations between the degree of WMH in frontal regions and the severity of depressive symptoms in the group of patients with $\mathrm{HF}$ and major depression.

Local Ethics Committees approved the study and written informed consent was obtained from all subjects. Eighteen patients with HF functional class II $(\mathrm{N}=12)$ or III (N =6) according to the New York Heart Association (FCNYHA) were recruited from the outpatient cardio-geriatric unit of the Instituto do Coração, Faculdade de Medicina, Universidade de São Paulo (InCor). The presence of HF was determined by detailed cardiological evaluation, including echocardiographic evidence of left ventricle ejection fraction below 50\%. All patients presented chronic symptoms of HF and had been stable on an optimized medication regimen for at least one year prior to the study. According to the diagnostic criteria of the Diagnostic and Statistical Manual of Mental Disorders, 4th edn. (DSM-IV), this sample of subjects with HF was then divided into a group of 10 non-depressed HF patients (FCNYHA-II = 7 and FCNYHA-III = 3) and a group of 8 
depressed HF subjects (FCNYHA-II $=5$ and FCNYHA-III $=3$ ). The diagnosis of major depressive disorder was based on the Structured Clinical Interview for DSM-IV (13). The mean age of the subjects at the onset of the current major depressive episode was 75.62 years $(\mathrm{SD}=7.35)$, and the mean duration of the depressive episode was 7.62 months $(\mathrm{SD}=5.44)$. Exclusion criteria for the two HF groups were: 1) presence of other medical conditions including neurological disorders, delirium and dementia; 2) use of any psychoactive medication in the last month; 3) history of myocardial infarction or cardiac surgery in the last 3 months; 4) diagnosis by DSM-IV criteria of major depressive disorder that had started before the diagnosis of HF, or the presence of psychotic symptoms (hallucinations and/or delusions); 5) diagnosis of other major psychiatric disorders; 6) family history of major depression or dementia; 7) presence of structural MRI findings compatible with brain stroke or other gross brain lesions or malformations.

A comparison group of 14 healthy elderly volunteers was recruited from the com- munity; they had no symptoms suggestive of physical, neurological or psychiatric disorders based on general medical questioning, physical and neurological examination and a Structured Clinical Interview for DSM-IV (13).

The demographic and clinical data for the three groups are given in Table 1. The groups were matched for age and years of education; however, they differed significantly in gender distribution (Table 1). As expected, there were significant differences between the overall HF sample and the healthy control group regarding left ventricle ejection fraction $(\mathrm{F}=132.76, \mathrm{P}<$ 0.001 ), and the presence of arterial hypertension $\left(\chi^{2}=10.71, \mathrm{P}=0.005\right)$. There were no significant differences between the two $\mathrm{HF}$ groups regarding the cause of $\mathrm{HF}$, the presence of arterial hypertension, or their FCNYHA (Table 1). Finally, a significant difference was observed between depressed HF patients and the two other groups in terms of the severity of depressive symptoms, as assessed quantitatively using the 31-item Hamilton scale (14). In the group of HF patients with major depression, all sub-

Table 1. Demographic and clinical variables of depressed and non-depressed subjects with heart failure and control subjects.

\begin{tabular}{lcccc}
\hline & Control (N=14) & HFnD (N=10) & HFD (N = 8) & P \\
\hline Age (SD) & $73.4(5.2)$ & $74.2(5)$ & $75.6(7.3)$ & $0.694^{* a}$ \\
Gender & & & & \\
$\quad$ Male (\%) & $4(28.6)$ & $8(80)$ & $3(37.5)$ & $0.037^{+a}$ \\
$\quad$ Female (\%) & $10(71.4)$ & $2(20)$ & $5(62.5)$ & \\
Mean number of years of education (SD) & $5.7(2.9)$ & $5.9(2)$ & $5.1(3.2)$ & $0.829^{\xi a}$ \\
Diabetes (\%) & $6(42.9)$ & $2(20.0)$ & $1(12.5)$ & $0.247^{+b}$ \\
Hypertension (\%) & $6(42.9)$ & $10(100)$ & $7(87.5)$ & $0.005^{+c}$ \\
Etiology of HF & & & & \\
$\quad$ Idiopathic (\%) & - & $5(50)$ & $4(50)$ & 1 \\
$\quad$ Ischemic (\%) & - & $5(50)$ & $4(50)$ & \\
Mean \%LVEF (SD) & $73.5(4.6)$ & $41.4(6.6)$ & $36(7.3)$ & $<0.001^{* b}$ \\
$\quad$ FCNYHA-II (\%) & - & $7(70)$ & $5(62.5)$ & $0.737^{+d}$ \\
$\quad$ FCNYHA-III (\%) & - & $3(30)$ & $3(37.5)$ & \\
Mean Hamilton Scores & $2.9(0.8)$ & $6.7(3.2)$ & $25.9(6)$ & $<0.001^{\xi b}$ \\
\hline
\end{tabular}

$\mathrm{SD}=$ standard deviation; $\mathrm{HFnD}=$ heart failure with no depression; $\mathrm{HFD}=$ heart failure with depression; $\mathrm{HF}=$ heart failure; \% LVEF = percentage of left ventricle ejection fraction; FCNYHA = functional class according to the New York Heart Association criteria.

${ }^{*} \mathrm{~F}_{\mathrm{a}}=0.37 ; \mathrm{F}_{\mathrm{b}}=132.76$ (ANOVA). ${ }^{+} \chi_{\mathrm{a}}^{2}=6.57 ; \chi_{\mathrm{b}}^{2}=2.8 ; \chi_{\mathrm{c}}^{2}=10.71 ; \chi_{\mathrm{d}}^{2}=0.11$ (exact Fisher test). ${ }^{\S} \mathrm{F}_{\mathrm{a}}=0.37 ;$ $\mathrm{F}_{\mathrm{b}}=24.73$ (Kruskal-Wallis test). 
jects had Hamilton scores higher than 18, which are in the range of moderate to severe depression.

MRI scans were acquired using a $1.5 \mathrm{~T}$ General Electric Signa LX CV scanner (Milwaukee, WI, USA). The presence of WMH was assessed on transaxial T2-weighted images acquired with the "fast spin-echo" technique (slice thickness of $6 \mathrm{~mm}$, interval between cuts of $0.6 \mathrm{~mm}$, head office $256 \times 256$, vision field $24 \times 24 \mathrm{~cm}$, echo train $=12$, repetition time $=4466 \mathrm{~ms}$, and echo time $=$ $98 \mathrm{~ms}$ ). Images were evaluated by a neuroradiologist with 14 years of research experience in brain MRI who was blind to the diagnostic status of all subjects, using the visual scale of Scheltens et al. (15). This instrument systematically assesses the localization, size and number of WMH in infraand supra-tentorial regions. Our radiologist has been involved in studies using this scale which demonstrated associations between the presence of $\mathrm{WMH}$ and a diagnosis of dementia (16). According to the criteria of the Scheltens scale, scores of periventricular regions range from 0 (absent) to 1 (hyperintensities up to $5 \mathrm{~mm}$ ) and 2 (from 6 to 10 $\mathrm{mm}$ ); in other brain regions, scores range from 0 (absent) to 1 (up to $3 \mathrm{~mm}$ and a maximum of 5 lesions), 2 (6 or more lesions up to $3 \mathrm{~mm}$ ), 3 (up to 5 lesions of 4 to 10 $\mathrm{mm}$ ), 5 (one lesion of more than $10 \mathrm{~mm}$ ) and 6 (confluent lesions).

First we investigated WMH in the frontal region according to our hypothesis using the variables: frontal periventricular WMH (PVWMH) and frontal deep WMH (D-WMH). Subsequently, we performed an exploratory investigation regarding the presence of WMH in the basal ganglia (caudate nucleus, putamen, globus pallidus, thalamus, and internal capsule), as well as in temporal, parietal, occipital, and cerebellar regions.

Using the SPSS software version 10 (SPSS Inc., Chicago, IL, USA), we used the nonparametric Kruskal-Wallis test and the Fisher exact test to compare all groups. Par- tial correlation coefficients between scores on the Hamilton and Scheltens scales in the depressed HF group were also calculated taking age as the confounding variable. The level of statistical significance was set at $\mathrm{P}<0.05$, one- tailed.

There were no significant differences in the frontal PV-WMH or frontal D-WMH between the three groups, either when the mean Scheltens scores were compared $(\mathrm{F}<$ $2.17, \mathrm{P}>0.34$ ) or when the percentage of subjects with each degree of severity was compared between groups $\left(\chi^{2}<8.35, \mathrm{P}>0.59\right)$.

In the depressed HF group, a significant positive partial correlation, controlling for age as a confounding variable, emerged between the severity of depressive symptoms and $\mathrm{WMH}$ ratings in the frontal $\mathrm{PV}-\mathrm{WMH}$ (partial correlation coefficient $=0.79, \mathrm{P}=$ 0.016 for both hemispheres; see Figure 1). The correlations between depression scores and frontal D-WMH were not statistically significant (partial correlation coefficient $=$ $0.51, \mathrm{P}=0.12$ for both hemispheres).

There were no significant between-group differences in WMH ratings in any of the other brain regions that were investigated exploratorily $(\mathrm{F}<4.5, \mathrm{P}>0.10$ for all regions). Also, no partial correlations between WMH ratings and Hamilton scores for de-

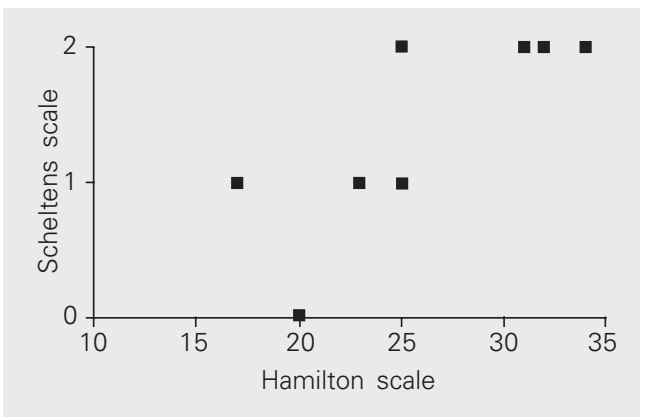

Figure 1. Distribution of scores on the Hamilton and Scheltens scales for subjects with heart failure and major depression for the frontal periventricular region. The correlation between severity of symptoms of depression and severity of scores on the Scheltens scale was statistically significant in this region (partial correlation coefficient $=0.79, P=0.016$, including age as a confounding covariate). Scores on the Scheltens scales were equal for both hemispheres. 
pression were obtained for these regions in the depressed HF group $(r<0.6, \mathrm{P}>0.06$ for all regions).

Our finding of a significant correlation between WMH and the severity of depressive symptoms in depressed HF patients, circumscribed to the frontal region, is compatible with the view that the emergence of depressive symptoms in association with HF may be related to vascular damage to white matter tracts connecting brain sites relevant to the regulation of mood (10). The fact that our significant correlation was restricted to $\mathrm{PV}-\mathrm{WMH}$ is consistent with studies on the general population that have related cardiovascular risk factors more consistently to PV-WMH $(10,17)$. On the other hand, other studies of clinical samples have shown that D-WMH may contribute more significantly to the presence of depressive symptoms in patients with stroke (6). Future studies with larger samples are needed to better distinguish the influence of D-WMH or PV-WMH on the severity of depressive symptoms in $\mathrm{HF}$ and related disorders.

An investigation of a large group of subjects with major depression has reported differences in WMH ratings depending on the presence of arterial hypertension, a highly relevant cardiovascular risk factor, although no mention was made of WMH ratings specifically in frontal regions (5). The fact that between-group differences in WMH in our study were not statistically significant may have been related to the presence of arterial hypertension in a fraction of the control subjects, as well as to the small size of our samples and the wide variability usually seen for scores on the Scheltens scale (15). In samples of HF subjects, the results of previous studies investigating $\mathrm{WMH}$ have been relatively inconsistent, reporting findings of either increases (7) or no differences (6) in WMH ratings in comparison to healthy subjects. However, those reports detected the presence of HF only by anamnesis (5), or using echocardiogram data in only some of the patients (6). Moreover, previous studies of WMH in HF patients have not reported data regarding the frequency of concomitant major depression. Our results suggest that the inconsistent results could be related to differences in the samples studied regarding the inclusion of patients with severe major depression.

Our results must be interpreted with caution, given the several limitations of the study. The fact that the groups were not gendermatched may have contributed to obscure WMH differences, as there have been reports of increased WMH incidence in males in the general population (18). However, the latter question is still under debate, and there have been reports of no WMH differences between sexes $(18,19)$. On the other hand, the association between WMH and advancing age is well established and this justified our strategy of matching groups for age and controlling the correlations between WMH and depression severity for the confounding influence of this demographic variable. Finally, the WMH was assessed by a single investigator using a visual inspection method, and this could cause difficulties in the replication of the results. Therefore, it is essential to extend our findings using automated methods for WMH evaluation in more representative samples of HF subjects. Nevertheless, it is interesting to mention that recent studies of WMH in elderly subjects have reported similar findings either with automated assessment methods or the visual method of Scheltens (20).

Our results provide preliminary evidence that the presence of frontal PV-WMH may be a contributing factor to the severity of major depressive symptoms in HF subjects. If confirmed in subsequent studies, these findings may also provide further support to the concept of "vascular depression", as well as stimulate studies aimed at developing broader strategies for the treatment and prevention of such vascular-related depressive conditions. 


\section{References}

1. Krishnan KR, Delong M, Kraemer H et al. (2002). Comorbidity of depression with other medical diseases in the elderly. Biological Psychiatry, 52: 559-588.

2. Fulop G, Strain JJ \& Stettin G (2003). Congestive heart failure and depression in older adults: clinical course and health services use 6 months after hospitalization. Psychosomatics, 44: 367-373.

3. Navarro V, Gasto C, Lomena F, Mateos JJ, Marcos T \& Portella MJ (2002). Normalization of frontal cerebral perfusion in remitted elderly major depression: a 12-month follow-up SPECT study. Neuroimage, 16 (Part 1): 781-787.

4. Thomas AJ, O'Brien JT, Davis S, Ballard C, Barber R, Kalaria RN \& Perry $\mathrm{RH}$ (2002). Ischemic basis for deep white matter hyperintensities in major depression: a neuropathological study. Archives of General Psychiatry, 59: 785-792.

5. Greenwald BS, Kramer-Ginsberg E, Krishnan KR, Hu J, Ashtari M, Wu H, Aupperle P, Patel M \& Pollack S (2001). A controlled study of $\mathrm{MRI}$ signal hyperintensities in older depressed patients with and without hypertension. Journal of the American Geriatric Society, 49: 1218-1225.

6. Longstreth Jr WT, Manolio TA, Arnold A, Burke GL, Bryan N, Jungreis CA, Enright PL, O'Leary D \& Fried L (1996). Clinical correlates of white matter findings on cranial magnetic resonance imaging of 3301 elderly people. The Cardiovascular Health Study. Stroke, 27: $1274-1282$.

7. Ylikoski A, Erkinjuntti T, Raininko R, Sarna S, Sulkava R \& Tilvis R (1995). White matter hyperintensities on MRI in the neurologically nondiseased elderly. Analysis of cohorts of consecutive subjects aged 55 to 85 years living at home. Stroke, 26: 1171-1177.

8. Steffens DC \& Krishnan KR (1998). Structural neuroimaging and mood disorders: recent findings, implications for classification, and future directions. Biological Psychiatry, 43: 705-712.

9. Alexopoulos GS, Kiosses DN, Choi SJ, Murphy CF \& Lim KO (2002). Frontal white matter microstructure and treatment response of latelife depression: a preliminary study. American Journal of Psychiatry, 159: 1929-1932.

10. Kumar A, Thomas A, Lavretsky H et al. (2002). Frontal white matter biochemical abnormalities in late-life major depression detected with proton magnetic resonance spectroscopy. American Journal of Psychiatry, 159: 630-636.

11. Krishnan KR, McDonald WM, Doraiswamy PM, Tupler LA, Husain M, Boyko OB, Figiel GS \& Ellinwood Jr EH (1993). Neuroanatomical substrates of depression in the elderly. European Archives of Psychiatry and Clinical Neuroscience, 243: 41-46.

12. Lee SH, Payne ME, Steffens DC, McQuoid DR, Lai TJ, Provenzale JM \& Krishnan KR (2003). Subcortical lesion severity and orbitofrontal cortex volume in geriatric depression. Biological Psychiatry, 54: 529-533.

13. First MB, Spitzer RL, Gibbon M \& Willians JBW (1995). Structured Clinical Interview for DSM-IV Axis I Disorders (SCID-I/P, version 2.0). Biometric Research Department, New York State Psychiatric Institute, New York.

14. Hamilton M (1960). A rating scale for depression. Journal of Neurology, Neurosurgery and Psychiatry, 23: 56-62.

15. Scheltens P, Barkhof F, Leys D, Pruvo JP, Nauta JJ, Vermersch P, Steinling M \& Valk J (1993). A semiquantitative rating scale for the assessment of signal hyperintensities on magnetic resonance imaging. Journal of the Neurological Sciences, 114: 7-12.

16. Campos ZMS (2001). White matter hyperintensities on magnetic resonance imaging in Alzheimer's disease and normal aging. Doctoral thesis, Instituto de Radiologia, Universidade de São Paulo, São Paulo, SP, Brazil.

17. Matsushita K, Kuriyama $Y$, Nagatsuka K, Nakamura M, Sawada T \& Omae T (1994). Periventricular white matter lucency and cerebral blood flow autoregulation in hypertensive patients. Hypertension, 23: $565-568$.

18. Coffey CE, Lucke JF, Saxton JA, Ratcliff G, Unitas LJ, Billig B \& Bryan RN (1998). Sex differences in brain aging: a quantitative magnetic resonance imaging study. Archives of Neurology, 55: 169179.

19. Allen JS, Damasio H, Grabowski TJ, Bruss J \& Zhang W (2003). Sexual dimorphism and asymmetries in the gray-white composition of the human cerebrum. Neuroimage, 18: 880-894.

20. Pantoni L, Simoni M, Pracucci G, Schmidt R, Barkhof F \& Inzitari D (2002). Visual rating scales for age-related white matter changes (leukoaraiosis): can the heterogeneity be reduced? Stroke, 33: 28272833. 